\title{
Unequal error protection for wireless data transmission using superposition coding with feedback
}

\begin{abstract}
High quality of data transmission services is an important task for wireless broadband communication systems. This paper is concerned with the design problem of real-time wireless data transmission which provides unequal error protection (UEP). Using superposition coding, the rea-time data stream is divided into two streams, high priority (HP) and low priority LP streams depending on their interest. These two bit streams are encoded separately, and then superimposed together with two different level of power to achieve the UEP for them in the receiver end. Feedback of channel state information (CSI) is used in adaptive physical layer channel for efficient use of the current available bandwidth. In this proposed scheme, same recourses; time, bandwidth and power are used to increase the transmission efficiency compared with other schemes which are use transmission of one data stream with one power level but in different techniques to exploit the available bandwidth.
\end{abstract}

